\title{
Columbus: aportes de la teledetección y análisis de coste anisotrópico a un debate histórico
}

\author{
Columbus: contributions of remote sensing and \\ anisotropic cost analysis to an historical debate
}

\author{
Ángel M. Felicísimo*
}

Recibido 05 de noviembre de 2019; aceptado 10 de diciembre de 2019

\section{Resumen}

La ruta seguida por la primera expedición de Cristóbal Colón es incierta debido a la ausencia o poca fiabilidad de esta información en el diario del almirante. Los actuales datos procedentes de teledetección y su análisis mediante SIG pueden aportar nueva información mediante análisis de los campos de viento estacionales en la zona. Los resultados del análisis de seis años muestran que el régimen de vientos es homogéneo y que configura dos corredores o pasillos de bajo coste relativo para la navegación a vela con las condiciones de los barcos del siglo XV. De estos dos corredores uno conduce con exactitud al destino al que se cree que llegó Colón el 12 de octubre de 1492 y se presenta como la ruta más probable, algo más al Sur que la propuesta hasta el presente en la bibliografía. Los SIG se muestran como una herramienta capaz de aportar información inédita a partir de datos globales, tomados en este caso por el escaterómetro QuikSCAT, aplicando métodos de análisis espacial poco usados en la literatura científica.

Palabras clave: Cristóbal Colón, análisis de coste anisotrópico, viento, QuikSCAT, Sistemas de Información Geográfica.

* Universidad de Extremadura, España, correo electrónico: amfeli@unex.es.

ORCID: https://orcid.org/0000-0002-9953-8614. 


\begin{abstract}
The route of the Christopher Columbus' first expedition is uncertain due to the absence or unreliability of this information in the admiral's diary. Current remote sensing data and GIS analysis can provide new information through analysis of the seasonal wind fields in the area. The results of the six-year analysis show that the wind regime is homogeneous and that it configures two relatively low-cost corridors for sailing with the conditions of 15 th century ships. One of these two corridors leads exactly to the destination where Columbus is believed to have arrived on October 12th 1492 and is presented as the most likely route, somewhat further south than that proposed so far in the literature. The GIS are shown to be a tool capable of providing unprecedented information from global data, taken in this case by the QuikSCAT catterometer, applying methods of spatial analysis rarely used in the scientific literature.

Key words: Christopher Columbus, anisotropic cost analysis, wind, QuikSCAT, Geographic Information Systems.
\end{abstract}

\title{
Introducción
}

La navegación marítima es una actividad humana que tiene varios miles de años de antigüedad. Las técnicas de navegación permitieron la colonización atravesando las barreras que suponían los océanos, antes absolutas. Partiendo de las balsas simples, apenas manejables, se consiguió un control progresivo de la navegación mediante la incorporación progresiva de elementos estructurales como remos, velas, diferentes formas de timón, etc. Algunas barcas ya ciertamente complejas se han datado en más de seis mil años en tumbas egipcias, aunque parece que se limitaban a la navegación fluvial. Estos elementos, el perfeccionamiento de los cascos y el aprendizaje sobre técnicas de orientación permitieron la navegación marítima a distancias más lejanas de las costas. Los fenicios dominaban el Mediterráneo en el primer milenio a.C. con barcos dotados de remos y vela, los cretenses tenían comercio marítimo mil años antes y la primera batalla naval documentada históricamente fue hacia el año 1200 a.C. entre chipriotas e hititas. En el otro lado del mundo, en los océanos Índico y Pacífico, la navegación oceánica permitió colonizar los archipiélagos de la Polinesia, cubriendo distancias de miles de km con canoas de uno o más cascos; por ejemplo, la cultura lapita llegó a Samoa y Tonga entre los años 1300 y 900 a.C. en una dispersión que les alejó hasta $6000 \mathrm{~km}$ desde su origen probable en el sudeste asiático. Debe mencionarse necesariamente la navegación vikinga que sobre el siglo IX llegó a Islandia, a Groenlandia en el siglo x y de la que se han encontrado restos de un asentamiento en L'Anse aux Meadows, en Terranova (McGovern, 1982).

Puede decirse que la edad de oro de la navegación oceánica fue en la segunda mitad del siglo XV, no solo con los viajes colombinos, sino con la apertura de rutas 
al Océano Índico por Bartolomeu Dias o Vasco de Gama, y la primera circunnavegación terrestre por la expedición Magallanes-Elcano que partió de España en 1519. Estos grandes viajes fueron posibles gracias a adelantos técnicos como la brújula - antiguo invento chino cuya primera mención en Europa es el siglo XII-, el astrolabio - conocido en el mundo islámico desde el siglo VIII y llegado a Europa hacia el XII-, o la corredera para estimar la velocidad - finales del siglo XV o inicios del XVI.

En este contexto, el objetivo del presente trabajo es presentar nueva información relativa a uno de los viajes marítimos más famosos de la historia: el de la expedición dirigida por Cristóbal Colón en 1492, que supuso el descubrimiento de América para el continente europeo. Esta información es el resultado de análisis de datos tomados por satélites y procesados mediante Sistemas de Información Geográfica (SIG) y es un ejemplo de cómo datos actuales pueden aportar algo de luz a acontecimientos pasados.

Este tipo de análisis ya fue utilizado por nuestro grupo de investigación en trabajos de naturaleza biológica sobre dispersión a larga distancia (Muñoz \& Felicísimo, 2006) y sobre migraciones de aves oceánicas (Felicisimo et al., 2008; Gonzalez-Solis et al., 2009) y ha conseguido aportar una evidencia sólida sobre el control del viento en los fenómenos de dispersión y migración a grandes distancias.

En este caso es necesaria una introducción sobre las características de la navegación en el siglo XV, las del primer viaje de Colón y las deficiencias en la documentación del mismo, derivadas de la ausencia de instrumentos para trazar la derrota de los barcos en aguas abiertas. Los datos siguientes se han tomado de Martín López (2019), que analiza y comenta la principal fuente disponible sobre los días del primer viaje colombino. Es el llamado "diario de a bordo de Colón", aunque en realidad es un manuscrito copia del original—o de una copia del original, actualmente, ambos perdidos__ realizada por Bartolomé de las Casas hacia 1530.

La primera cuestión relevante para este trabajo es que no se conocen con detalle las características concretas de los barcos que se usaron en la expedición. Aunque suele hablarse de tres carabelas, de nombres "Santa María", "Pinta" y "Niña", la opinión más aceptada en estos momentos es que Colón armó una nao, la "Santa María", nave capitana, y dos carabelas: la "Pinta" y la "Niña". El aspecto más importante de esta cuestión es que la nao era una embarcación incapaz de navegar con vientos laterales por lo que su rumbo era esencialmente el del viento en popa, con ángulos de ceñida muy cerrados. La carabela tenía más capacidad en la navegación aprovechando el viento lateral, pero se sabe por el mencionado diario de Colón que los tres barcos fueron juntos, por lo que los rumbos cortando el viento estaban limitados a las capacidades de la nao Santa María.

La segunda cuestión es sobre la derrota de los barcos y, en concreto, sobre la determinación de longitud, latitud y distancia recorrida. Ya se ha comentado las limitaciones de los instrumentos en esta época, pero a esto se añade la escasez y poca 
fiabilidad de los datos que Colón incluye en su diario. Hay numerosas referencias al uso de la brújula, pero Martín López (2019) señala las grandes dificultades para mantener el rumbo algo que "solo era posible realizar con viento favorable y de dirección constante". Por otra parte, no hay datos de latitud ni longitud. Sobre la longitud no hay sorpresa ya que su determinación con cierta fiabilidad solo fue posible varios siglos más tarde, con la disponibilidad de cronómetros suficientemente exactos. La latitud podría haberse determinado con el cuadrante —el sextante no existía - pero solo se mencionan medidas en tres ocasiones y en todas ellas los datos se consideran pésimos, hasta el extremo de que Colón decidió dejar de tomarlos.

Finalmente, la distancia recorrida es prácticamente una incógnita ya que la corredera no se había inventado aún y, aunque hay estimaciones en el diario, su fiabilidad es muy dudosa. Por añadidura, Colón reconoce en su diario que miente sistemáticamente para no alarmar a las tripulaciones por las grandes distancias recorridas.

A partir de esta información, se han planeado hipótesis sobre la ruta de esta primera expedición. En la Figura 1 se puede ver la trayectoria mostrada en un mapa de la Biblioteca Virtual Miguel de Cervantes (Fundación Biblioteca Virtual Miguel de Cervantes, s.f.) donde el viaje transcurre desde La Gomera $\left(28^{\circ} \mathrm{N}\right)$ hasta arribar al supuesto punto de llegada, la isla de San Salvador $\left(24^{\circ} \mathrm{N}\right)$.

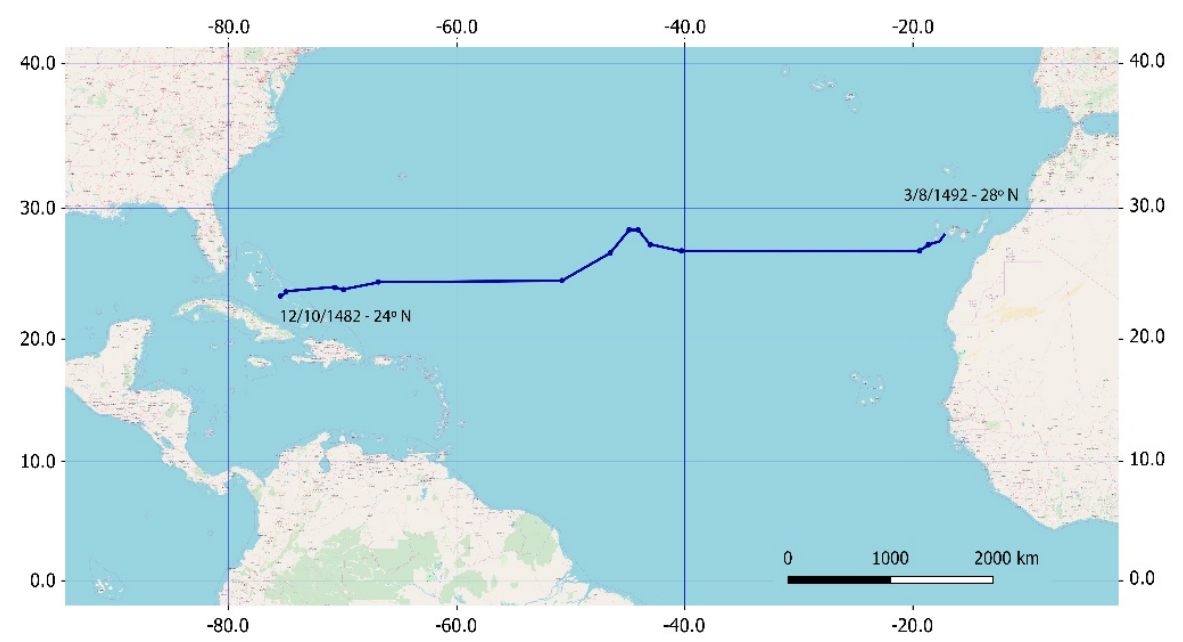

Figura 1. Trayectoria del primer viaje a América de Cristóbal Colón.

Fuente: Elaboración propia a partir del mapa de la Biblioteca Virtual Miguel de Cervantes; base OSM.

En resumen, la trayectoria o derrota de las naves es incierta y, aunque hay hipótesis diversas, la verificación de las mismas no es posible con los datos disponibles. Los datos de los que partimos en este trabajo son pocos pero suficientes para obtener 
algunos resultados: 1) el punto de salida de las naves fue la isla de La Gomera, en las Islas Canarias y 2) las naves no tenían capacidad para realizar ceñidas significativas y debían mantener el viento por la popa con pocas desviaciones.

A partir de estas premisas, la idea del presente trabajo es realizar simulaciones de trayectorias sobre campos de viento en la misma zona y en las mismas fechas. El objetivo es mostrar la ruta más probable, compatible con las restricciones de entrada. Para ello es necesario disponer de datos de viento y de métodos de análisis que muestren la mayor o menor dificultad para desplazarse sobre ellos en función de su acimut y velocidad. El método adecuado para esta labor se denomina análisis de coste anisotrópico y será explicado posteriormente.

\section{Material y métodos}

\section{Sistemas de Información Geográfica}

El análisis de coste anisotrópico se ha realizado con el Sistema de Información Geográfica ArcInfo (ESRI Inc.). El motivo es que cuando comenzamos a trabajar con este tipo de análisis, existían varias opciones para trabajar con análisis de coste isotrópico, pero solo ArcInfo tenía herramientas para el anisotrópico. Durante los primeros trabajos, comenzados en el año 2003, se desarrolló y depuró una cantidad notable de código para este tipo de procesamiento. Aunque parte de él ha cambiado, especialmente el de lectura y transformación de los ficheros originales, lo hemos conservado durante más de una década completamente funcional, aunque hace unos años que dejó de comercializarse. Las rutinas fueron programadas en el lenguaje propio de ArcInfo, AML y, con modificaciones menores, son las que se han utilizado para este trabajo.

La generación de mapas e imágenes y algunos cálculos de asignación de valores de coste a localizaciones se ha realizado con QGIS 3.10.

\section{Datos de viento}

Los datos de viento provienen del escaterómetro SeaWinds portado por el satélite QuikSCAT, desarrollado por el JPL de la NASA para medir la velocidad y la dirección de los vientos de la superficie del océano. El primer QuikSCAT fue lanzado el 19 de junio de 1999 y posteriormente se han sucedido varios satélites portadores de instrumentos similares ${ }^{1}$. El instrumento SeaWinds es un radar especializado (escaterómetro) que mide la velocidad y dirección de los vientos a una altura nominal de $10 \mathrm{~m}$ sobre la superficie marina (JPL, Jet Propulsion Laboratory, 2001).

Todos los productos estándar de QuikSCAT están en formato HDF. Los archivos HDF de QuikSCAT contienen un conjunto de 16 SDS - scientific datasets-; cada 
uno de ellos es una matriz rectangular de 1440 píxeles de este a oeste y 720 píxeles de norte a sur $0.25^{\circ}$ en una proyección estándar "plate carrée" con $0.25^{\circ}$ de resolución espacial en el Ecuador.

Para este trabajo se han usado datos de los años 1999 a 2005 correspondientes a los períodos que coinciden con la expedición colombina. Los datos descargaron del PO-DAAC -Physical Oceanography Distributed Active Archive Center) donde están disponibles gratuitamente ${ }^{2}$. Los años más recientes han sido descartados para evitar la posible influencia de los últimos años muy cálidos en los patrones espaciales de trayectorias del viento, algo no verificado pero potencialmente posible.

El procesamiento de este tipo de datos no es inmediato. Los valores de una imagen clásica de teledetección son datos escalares, distribuidos en una escala lineal, que pueden ser procesados por métodos estadísticos convencionales. Sin embargo, los píxeles del QuikSCAT almacenan información vectorial ya que representan velocidad y acimut del viento mediante los componentes $u$ y $v$ de cada vector, proyecciones de cada vector sobre los ejes X e Y, cada uno en un SDS diferente. De acuerdo con la convención adoptada para los datos de QuikSCAT, una dirección de viento de $0^{\circ}$ implica un flujo hacia — no desde- el Norte.

El primer paso en el procesamiento de los vectores QuikSCAT para este trabajo ha sido la extracción de los SDS que contienen los valores $u$ y $v$ de dichos vectores $\mathrm{y}$ su transformación a un formato utilizable por el SIG utilizado que en este caso fue ESRI grid.

El procesamiento de este tipo de datos en un SIG, presenta algunas dificultades que es necesario superar. La primera de ellas es que la cobertura diaria de QuikSCAT no es completa, efecto de la trayectoria orbital. Las áreas sin datos no pueden aceptarse porque rompen la continuidad espacial necesaria para el análisis de la trayectoria anisotrópica. Como esas áreas vacías cambian diariamente, la solución adoptada fue generar una síntesis de los días de duración del viaje calculando la media de los valores de $u$ y $v$, aprovechando que los vientos son muy constantes en estas latitudes. Tras este proceso, se calcularon los componentes acimut y módulo para cada año ya que son entradas necesarias para el cálculo de coste.

La segunda dificultad es que, aunque todos los SIG implementan opciones para realizar álgebra de mapas, los vectores de viento no pueden ser analizados con estadística ni operaciones convencionales porque están distribuidos en una escala circular. Parte del código desarrollado lo fue para poder operar con este tipo de datos y poder aplicar estadística y operaciones específicas para datos circulares (Fisher, 1995; Mardia, 1972). Estos procedimientos incluyen rutinas para la correcta proyección del acimut en función de la proyección que, en este caso, dada la zona de trabajo 
y las propiedades que deben analizarse, ha sido una Mercator, cilíndrica y tangente al Ecuador.

\section{Análisis de coste anisotrópico}

El análisis de coste anisotrópico es un procedimiento que estima el coste acumulativo de moverse desde una celda origen determinada a cada una de las otras celdas en un modelo raster. El nuestro caso se analiza el coste de moverse sobre una superficie de fricción determinada por los campos de viento — velocidad y acimut en cada tesela o celda de la superficie. El coste de moverse con un acimut determinado — por ejemplo, hacia el oeste - sobre una celda es el inverso de la velocidad del viento multiplicada por un "factor horizontal" que penaliza progresivamente las desviaciones angulares respecto al acimut del viento. En el caso de los barcos, a igualdad de velocidad del viento, el menor coste es cuando se mueven con viento de popa y el mayor con viento de proa.

Una vez disponibles los valores de acimut y velocidad en cada celda, el análisis de coste anisotrópico se desarrolla definiendo una celda origen (isla de La Gomera) a partir de la cual construye una superficie de coste donde se refleja el coste acumulado mínimo para llegar a cada celda del modelo desde la celda origen. Este proceso supone un análisis completo de las rutas potenciales hasta cada celda desde el origen, descartándolas todas salvo la que presente el valor de coste acumulado mínimo. Este procedimiento se ha realizado para cada año del periodo estudiado.

\section{Síntesis de costes en los puntos de llegada potenciales}

La parte final del análisis ha sido la valoración del coste acumulado para una serie de puntos de llegada alternativos o potenciales. Para ello se ha distribuido una cadena de puntos cercanos a las costas de América y asignado a cada uno el coste acumulado calculado en la fase anterior. La visualización conjunta de estas cadenas (una por año) permite valorar la homogeneidad o heterogeneidad de los resultados y la localización, en su caso, de "pasajes" de bajo coste relativo, donde los barcos hubieran podido llegar con más facilidad en el escenario de los campos de viento de cada año. Todo ello se refleja en los mapas de síntesis finales.

\section{Resultados}

\section{Datos de entrada}

Los datos de entrada para el análisis de coste anisotrópico son, como se ha mencionado en el apartado anterior, los modelos raster de velocidad y acimut del viento. La Figura 2 muestra un ejemplo de la distribución de la velocidad del viento en la zona correspondiente al año 1999. El resto de años muestra una limitada variabilidad como se podrá ver en los mapas finales. 


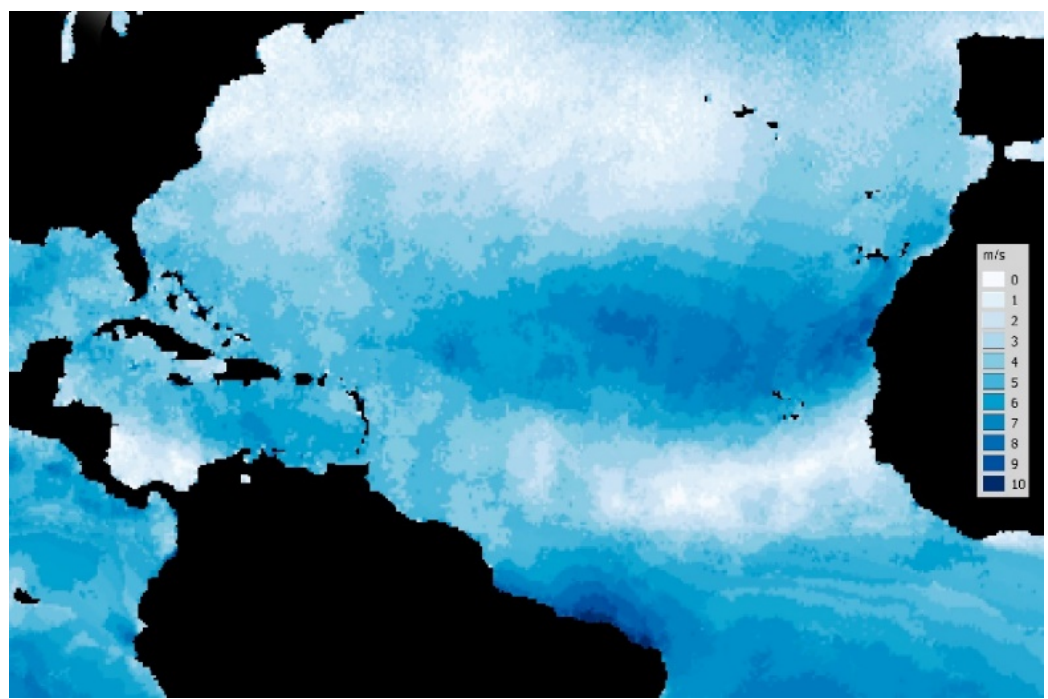

Figura 2. Velocidad media del viento en el periodo de estudio del año 1999.

La Figura 3 muestra la distribución de acimutes del viento para el mismo año 1999. Dado que es una variable de distribución circular, hay una discontinuidad en la codificación de tonos entre los 359 y los $0^{\circ}$. Se dispone de estos modelos para cada año del periodo 1999-2005; en ellos, la tierra emergida tiene valor nodata y actúa como barrera de resistencia infinita.

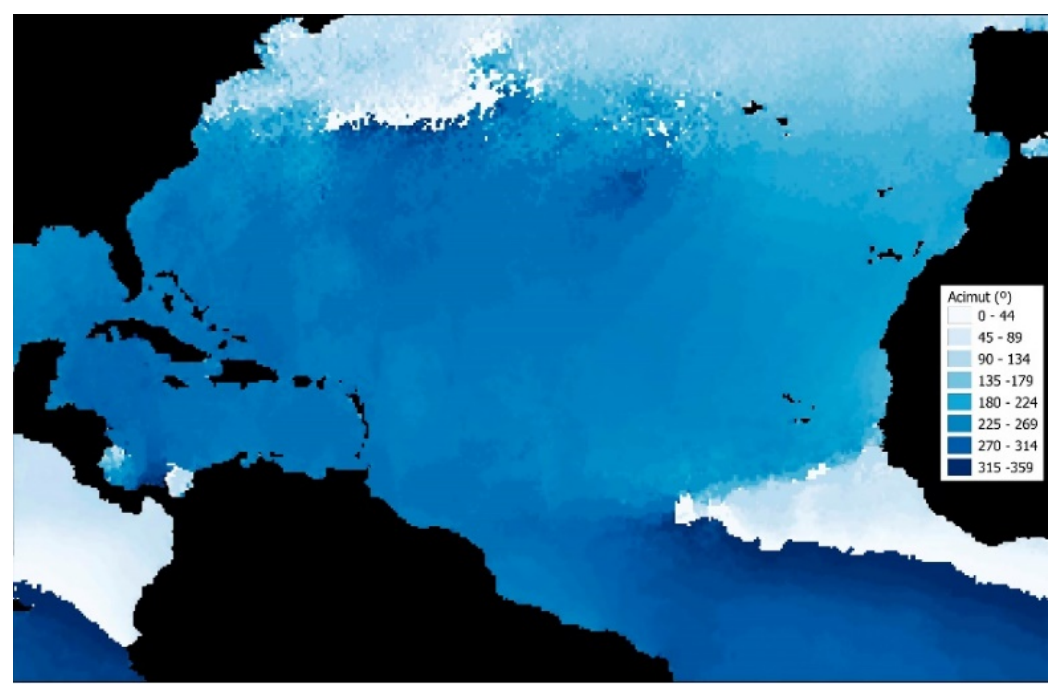

Figura 3. Acimutes del viento en el periodo de estudio del año 1999. 


\section{Coste acumulado y ventanas de llegada}

Los resultados finales se reflejan mediante mapas de coste acumulado con punto de origen en la isla de La Gomera. La Figura 4 muestra ese modelo para el año 1999. En cada modelo se ha añadido una cadena de puntos cercanos a la costa americana para los cuales se ha transferido el valor de coste de la celda correspondiente y codificado el resultado en colores. El objetivo es ver con más claridad lo que podríamos llamar "ventanas" o zonas de arribada preferentes, con mínimos de coste que suponen una llegada más probable a esa zona de costa si sólo actuara el viento. En la Figura 5 se muestra los resultados para todos los años analizados y la media aritmética.

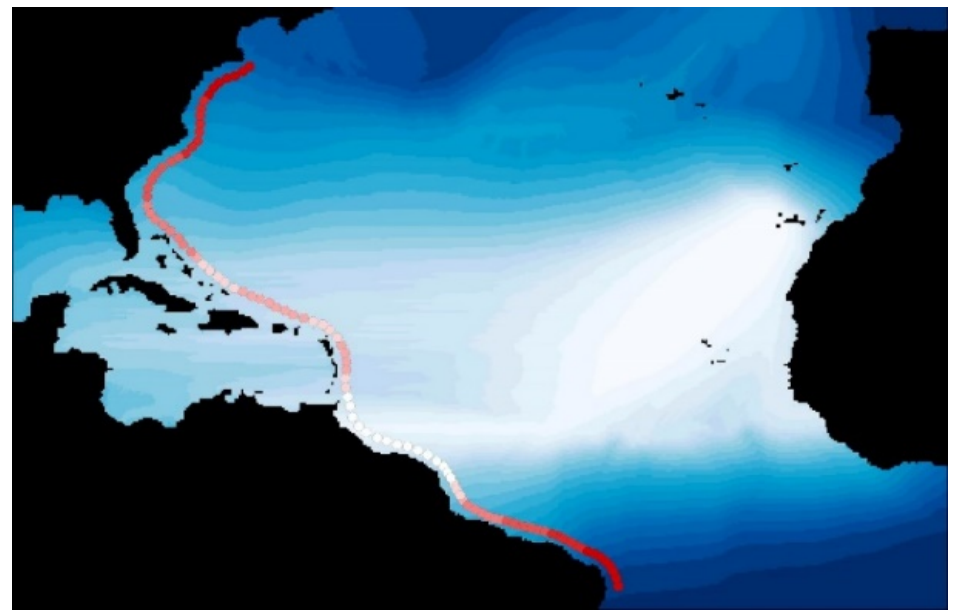

Figura 4. Modelo de coste y "ventanas" de llegada para el año 1999. Las zonas de llegada más probables en función del viento son los círculos de tonos más claros.
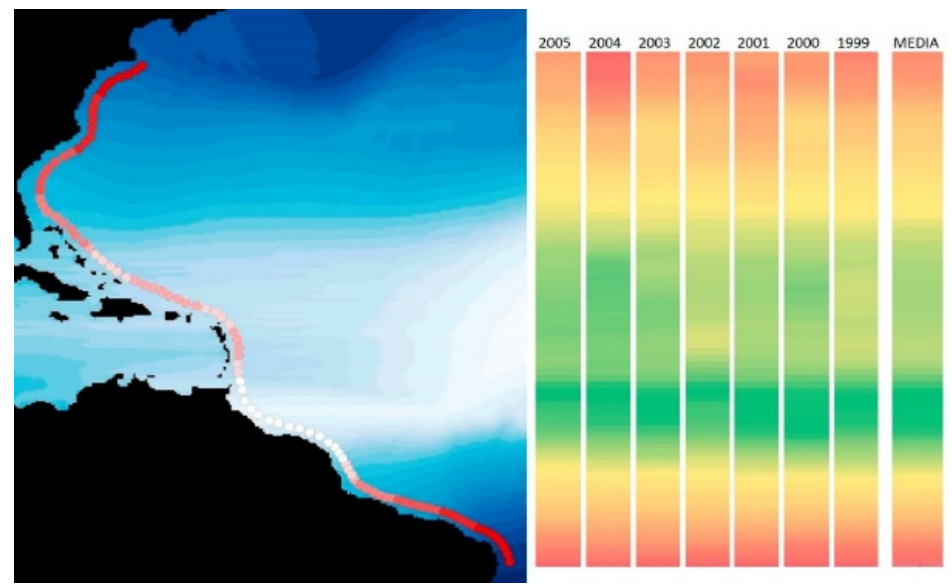

Figura 5. Coste acumulado em diferente puntos de llegada a las costa americana para los años 1999 a 2005 y su media aritmética. Las zonas en verde son la "ventanas" de llegada de mínimo coste. 
Finalmente, se han trazado los caminos de mínimo coste a cada punto de la cadena para reflejar si las rutas siguen un mismo trazado o si hay alternativas diferentes en función del viento; la Figura 6 muestra los resultados.

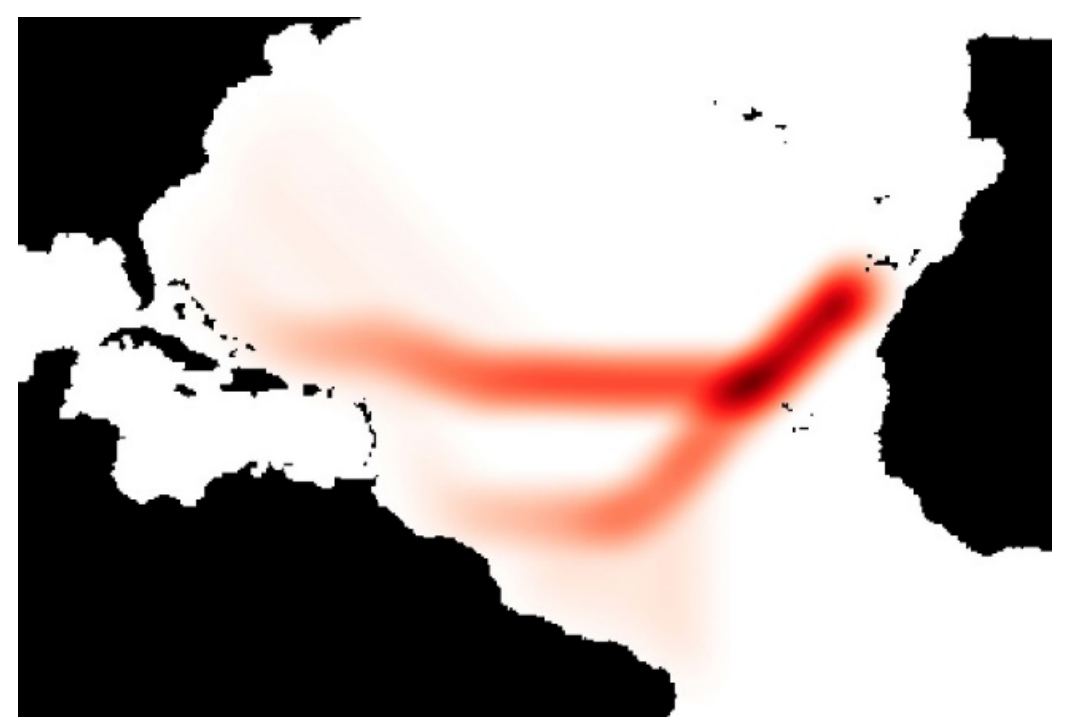

Figura 6. Caminos de coste mínimo para la llegada a las costas de América con la isla de La Gomera como punto de origen.

\section{Discusión}

Los resultados anteriores muestran varias cuestiones de interés para el tema de debate: la ruta de Colón en su viaje a América. La primera es que los caminos posibles están limitados para naves cuya capacidad de viajar con el viento de costado era muy limitada. Si se asume esta limitación, los modelos de coste utilizados asignan al océano los costes que definen las rutas potenciales menos costosas para una embarcación que solo dependiera del viento como fuerza de impulsión (Figura 4). La segunda es que los resultados en los años analizados son bastante homogéneos (Figura 5), se muestran dos "ventanas" de llegada a latitudes diferentes. La más estable se sitúa sobre los 4 y $10^{\circ}$ de latitud norte, por encima de la Zona de Convergencia Intertropical y entre la Guayana Francesa y Trinidad y Tobago. La segunda, menos intensa y con algo más de variabilidad interanual, está sobre los 22 a $25^{\circ} \mathrm{N}$, en la zona entre Islas Turcas y Caicos y Bahamas.El cálculo explícito de los caminos de mínimo coste hacia América permite elaborar la Figura 6, se observan dos rutas alternativas que podrían haberse seguido en función de las limitaciones de la navegación asumidas. Igualmente se muestra que a la salida de La Gomera, el rumbo más adecuado es el SO y que, llegando a las latitudes de las Islas de Cabo Verde, se presentan dos 
alternativas: seguir hacia el SO o virar directamente hacia el oeste y atravesar el Atlántico sin variar este rumbo. En las escasas anotaciones de su diario, Colón mantuvo que su rumbo era hacia el oeste por lo que en este escenario, la expedición colombina debió tomar la primera ruta de estas dos alternativas. No se menciona, sin embargo, las primeras etapas hacia el SO que en el contexto del coste, serían prácticamente obligadas. En cualquier caso, la ruta directa al Oeste, tomada desde La Gomera, aparece improbable en este análisis dada la constancia de vientos al SO y la gran extensión de la zona de calmas o vientos débiles que se extiende al oeste de las Islas Canarias (Figura 2). Cabe mencionar un trágico ejemplo de la segunda ruta hacia las costas de Venezuela: en mayo del año 2006, se encontró una embarcación con una docena de cadáveres a bordo que intentó llegar desde las costas de África a probablemente, las Islas Canarias, un camino de inmigración a Europa relativamente común. Tras quedar a la deriva, fueron arrastrados por el viento y las corrientes durante días, fueron encontrados a unas 80 millas al Sur de Barbados, exactamente en el lugar de llegada de la segunda ruta mostrada en este trabajo.

Queda finalmente por comentar que el punto de llegada de la expedición colombina se ha situado en la isla de San Salvador, en las Bahamas, que con su latitud de $24^{\circ} \mathrm{N}$ queda exactamente en la salida de la primera ruta mostrada en la Figura 6.

En conclusión, en este trabajo se muestra un análisis que utilizando datos de un escaterómetro portado por el satélite QuikScat y cálculos de coste sobre los campos de viento oceánicos, aporta información sobre la ruta posible de la primera expedición de Colón, apoyando la hipótesis de una ruta latitudinal relativamente alta, pero no la mostrada en la Figura 1 ya que se incluiría una primera etapa con rumbo SO que, con los datos actuales, es prácticamente obligada para los barcos de la época. Este trabajo puede servir como ejemplo de utilidad de este tipo de análisis, ya demostrada en trabajos previos en otros contextos (véase la "Introducción") aunque, lógicamente, no es una prueba definitiva que pueda resolver la incógnita que se aborda. El principal problema es que los vientos pudieron haber cambiado en cinco siglos y hacer inaplicables los resultados actuales. Es cierto que el régimen general de los alisios en el Atlántico Norte ya es conocido y se usa en la navegación en los siglos siguientes a este viaje y también es conocido que la ruta de vuelta fue, coherentemente con el régimen general de vientos en la zona, por latitudes mucho más elevadas. No puede descartarse sin embargo, variaciones locales o temporales que desplazarían la ruta real de los barcos respecto a las modelizadas en este análisis pero la uniformidad de los años estudiados aporta cierta garantía a la solidez del resultado.

\section{Bibliografía}

Felicísimo, A.M., Munoz, J. \& Gonzalez-Solis, J. (2008). "Ocean Surface Winds Drive Dynamics of Transoceanic Aerial Movements", en Plos One, 3(8). https://doi.org/10.1371/journal.pone.0002928 
Fisher, N.I. (1995). Statistical analysis of circular data (Repr. 1. paperback ed). Univ. Press.

Fundación Biblioteca Virtual Miguel de Cervantes (s. f.). Cristóbal Colón en la Biblioteca Virtual Miguel de Cervantes. Biblioteca Virtual Miguel de Cervantes. Recuperado 18 de enero de 2020. Recuperado de http://www.cervantesvirtual.com/portales/cristobal colon/

Gonzalez-Solis, J., Felicísimo, A., Fox, J.W., Afanasyev, V., Kolbeinsson, Y. \& Munoz, J. (2009). "Influence of sea surface winds on shearwater migration detours", Marine Ecology Progress Series, no. 391, pp. 221-230. https://doi.org/10.3354/meps08128

JPL, Jet Propulsion Laboratory, J. (2001). QuikSCAT Science Data Product. User's Manual. Version 2.2 (D-18053; p. 95). JPL, Jet Propulsion Laboratory. ftp://ftp.scp.byu.edu/docs/ProjDocs/QSUG4-4.pdf

Mardia, K.V. (1972). Statistics of directional data, Academic Press.

Martín López, J. (2019). El viaje del Descubrimiento. Notas y comentarios al diario de Colón, 2a ed., Centro Nacional de Información Geográfica. Recuperado de http://www.ign.es/web/resources/acercaDe/libDigPub/ViajeDescubrimiento.zip

McGovern, T.H. (1982). "The Vinland Adventure: A North Atlantic Perspective", North American Archaeologist, 2(4). Recuperado de https://journals.sagepub.com/doi/abs/10.2190/6HN5-AC9T-TWVJ-QDXM

Muñoz, J. \& Felicísimo, A.M. (2006). Wind as a long distance dispersal vehicle in the Southern Hemisphere. XVII International Botanical Congress Abstracts, XVII International Botanical Congress Abstracts, 74. 